\title{
In situ observations on deformation behavior and stretching- induced failure of fine pitch stretchable interconnect
}

\author{
Yung-Yu Hsu ${ }^{\text {a) }}$ \\ IMEC, Kapeldreef 75, 3001, Leuven, Belgium; and Department of Materials Engineering, \\ Katholieke Universiteit Leuven, 3000 Belgium \\ Mario Gonzalez \\ IMEC, Kapeldreef 75, 3001, Leuven, Belgium \\ Frederick Bossuyt, Fabrice Axisa, and Jan Vanfleteren \\ IMEC-Centre for Microsystems Technology, 9052 Gent-Zwijnaarde, Belgium \\ Ingrid De Wolf \\ IMEC, Kapeldreef 75, 3001, Leuven, Belgium; and Department of Materials Engineering, \\ Katholieke Universiteit Leuven, 3000 Belgium
}

(Received 15 June 2009; accepted 29 July 2009)

\begin{abstract}
Electronic devices capable of performing in extreme mechanical conditions such as stretching, bending, or twisting will improve biomedical and wearable systems. The required capabilities cannot be achieved with conventional building geometries, because of structural rigidity and lack of mechanical stretchability. In this article, a zigzag-patterned structure representing a stretchable interconnect is presented as a promising type of building block. In situ experimental observations on the deformed interconnect are correlated with numerical analysis, providing an understanding of the deformation and failure mechanisms. The experimental results demonstrate that the zigzag-patterned interconnect enables stretchability up to $60 \%$ without rupture. This stretchability is accommodated by in-plane rotation of arms and out-of-plane deformation of crests. Numerical analysis shows that the dominating failure cause is interfacial in-plane shear stress. The plastic strain concentration at the arms close to the crests, obtained by numerical simulation, agrees well with the failure location observed in the experiment.
\end{abstract}

\section{INTRODUCTION}

Large area deformable macroelectronics have attracted increasing attention in recent years, particularly because they cannot only withstand bending and twisting but also the most challenging deformations, such as stretching. This deformability allows the macroelectronics to be used in applications that are hard to cover by conventional semiconductor microelectronics. ${ }^{1,2}$ Therefore, various concepts have been developed in recent years to fulfill the demands of extreme deformations. For most of these concepts, thin and lightweight sheets of elastic polymers represent ideal substrates. By depositing conductive polymers or small molecule organics on top of these substrates, it is believed that flexibility and modest stretchability can be achieved. However, the main disadvantage of this concept is that the electrical performances are often limited by the material properties of the polymers or molecules used. For example, the

\footnotetext{
a) Address all correspondence to this author.

e-mail: hsu@imec.be

DOI: 10.1557/JMR.2009.0447
}

mobility of polymers and molecules is weaker than those composed of inorganic materials. This consideration, combined with the uncertain reliability of the organics, has recently led to interest in using hybrid systems. These hybrid systems are usually composed of inorganic-based rigid or bendable elements and an organic-stretchable substrate such as rubber. To achieve deformability, the rigid or bendable inorganic-based elements are connected by stretchable conductors on or in the stretchable substrate. This hybrid system concept can be applied in biomedical systems that require high reliability and performance. Because the thin conductor lines that connect the active components have to withstand deformations, a proper structural design is essential to avoid losing structural integrity and electrical functionality during extreme deformations.

Several technologies for stretchable conductors have been proposed in recent years. For example, by designing thin film with a wavy or buckling period, adhered on polymer substrate, ${ }^{3,4}$ the level of stretchability can be controlled. More recently, this wavy thin film technology has been applied in stretchable systems, such as 
photodetectors ${ }^{5}$ and oscillators. ${ }^{6,7}$ A similar technology involving spontaneous wrinkling metal films ${ }^{8-10}$ has also been studied. However, microcracking often plays an important role in the mechanics of stretching of such films. With microcracking in wrinkling metal film, the electrical resistance changes with elongation. This unstable electrical property is certainly a disadvantage of a stretchable conductor for certain applications.

An alternative technology involves in-plane patterned metal conductors. For example, sinusoidal shaped thin metal film deposited on an elastomeric substrate has been studied through parametric analysis. ${ }^{11}$ It is also reported that the horseshoe-shaped conductors ${ }^{12-14}$ can be stretched up to $100 \%$ without material rupture. Another simpler example for an in-plane patterned metal conductor is the zigzag structure. ${ }^{15}$ The advantage of the zigzag structure above the horseshoe is that it can be applied in a high $\mathrm{I} / \mathrm{O}$ density device while maintaining high stretchability and stable electrical resistance. With a proper structural design, the metal interconnect acts like a spring while stretching, even though the intrinsic fracture limit of the material is at strains of $1 \sim 2 \%{ }^{16} \mathrm{By}$ applying these in-plane patterned metal conductors as interconnects, a hybrid system can reach high stretchability without losing its electrical performance. In other words, the performance of a stretchable system is the same as that of a conventional bulk system. In addition, this attractive coplanar technology is easy to use in current conventional planar microfabrication technology without requiring many processing modifications. This approach allows a cost reduction, which is one of the major concerns in industry.

In this article, we comprehensively investigate the deformation behavior of a zigzag structure during stretching by using both experimental observations and numerical analysis. Analytical models are developed to understand the mechanical relationship between the stress induced by external loading and deformability of the zigzag interconnect. To study the failure mechanics, the in situ observations of stretching zigzag interconnects are correlated to numerical analysis. These observations and numerical analysis explain all failure mechanisms. By combining analytical, numerical, and experimental analysis, design guidelines for future improvement on the mechanical robustness can be provided.

\section{NUMERICAL MODELING}

Figure 1 illustrates the patterned zigzag metal conductor design adhered on an elastomeric substrate. For visual clarification, only five crests are shown. The angle $(\theta)$ of each crest of the patterned zigzag metal conductor is 60 degrees. The width $\left(w_{\mathrm{Cu}}\right)$, thickness $\left(t_{\mathrm{Cu}}\right)$, and length $\left(l_{\mathrm{Cu}}\right)$ of the metal track are $0.1,0.018$, and $2 \mathrm{~mm}$, respectively. The substrate is a block with $W_{\text {sub }}=$

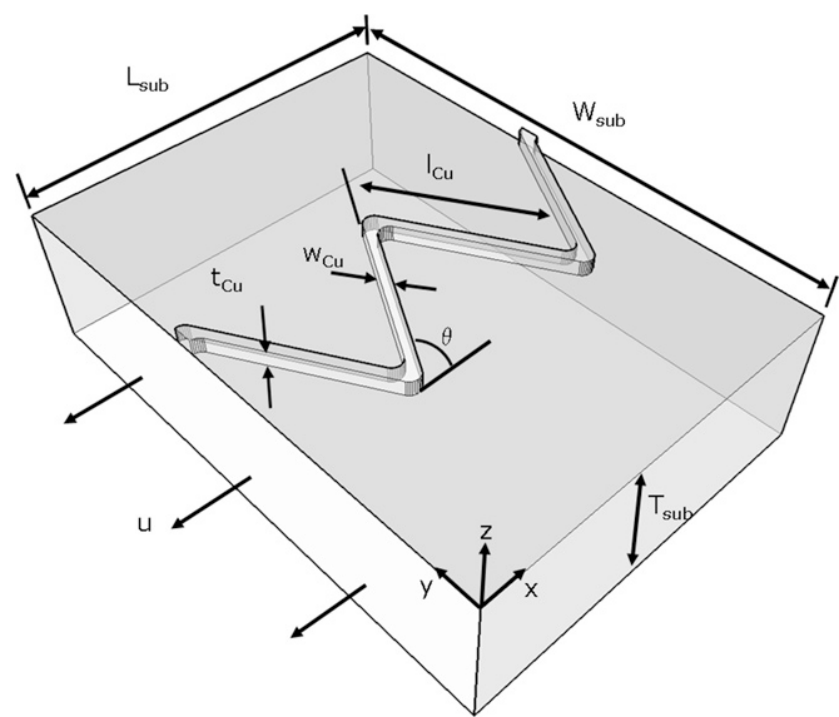

FIG. 1. Schematic illustration of patterned metallic zigzag interconnection on top of PDMS substrate.

10-mm wide, $L_{\text {sub }}=70-\mathrm{mm}$ long, and $T_{\text {sub }}=0.5-\mathrm{mm}$ thick. A uniaxial elongation " $u$ " is applied to the substrate on the two end surfaces as the boundary condition, which corresponds to the experimental conditions. The commercial finite element code, MSC.MARC (Santa Ana, CA), was used to simulate the deformation process of the patterned metal on an elastomeric substrate. The metal used in this research is copper and is modeled as a plastically deformable solid, obeying the bi-linear kinematic hardening rule, with the elastic Young's module $E_{0}=117 \mathrm{GPa}$, the yielding point at $\sigma_{\mathrm{y}}=0.1723 \mathrm{GPa}$, and the tangent module $E_{\mathrm{t}}=1.0342 \mathrm{GPa}$. The elastomeric substrate [poly-dimethyl siloxane (PDMS)] is modeled as an incompressible hyperelastic Neo-Hookean solid ${ }^{17}$ with $\mathrm{C}_{10}=0.157$.

\section{ANALYTICAL MODEL OF ZIGZAG INTERCONNECT UPON STRETCHING}

To have a "first view" on the stress distribution and to determine the relationship between the applied loading and deformation of the zigzag structure, we first used an energy method to calculate the strain energy in the zigzag structure under applied loading, $P$, which is kept along the $x$-direction. The substrate is not taking into consideration in our analytical model. Thus, the transversal compressive loading induced by Poisson ratio of the substrate is not included in the analysis. The zigzag structure is divided into unit sections, each containing one arm only, as shown in Fig. 2(a). The total strain energy in each unit section consists of two parts, the longitudinal energy $U_{l}$ due to the longitudinal tensile loading along the arm, and the bending energy $U_{M}$ perpendicular to the arm. We begin with a model based on 


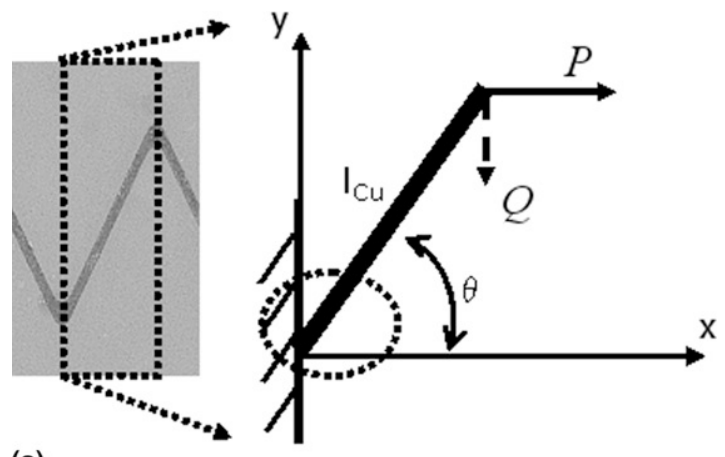

(a)
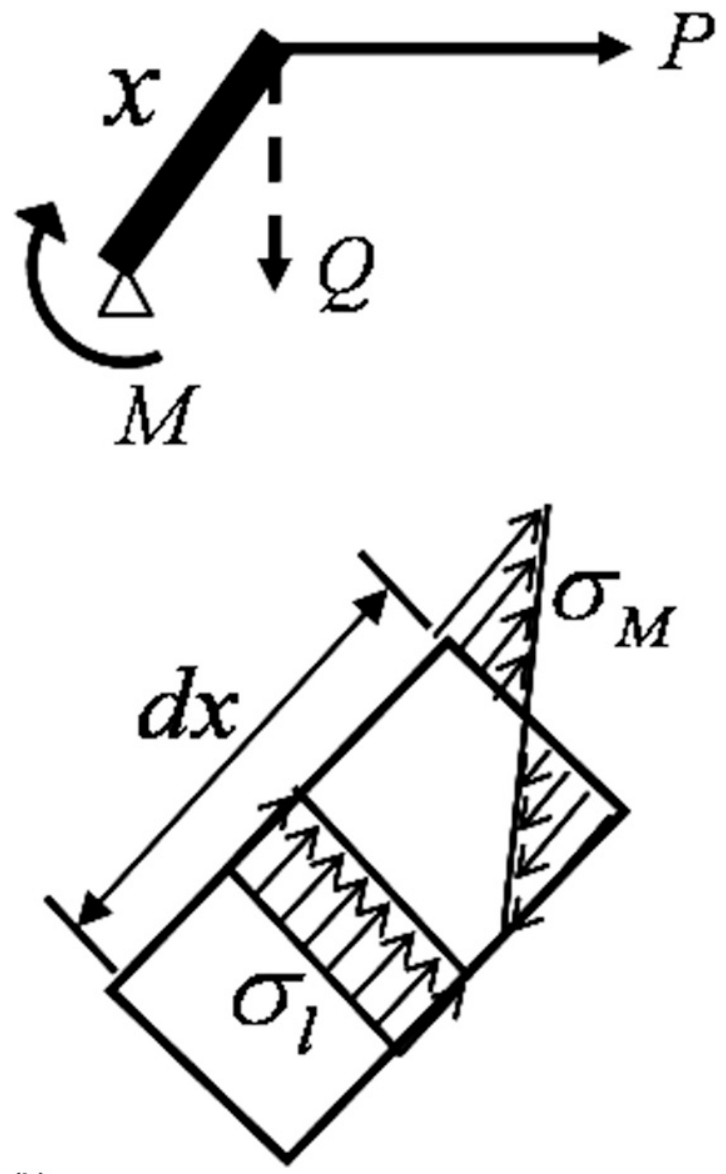

(b)

FIG. 2. (a) Schematic illustration for analytical analysis. (b) Freebody diagram.

Hook's law. Therefore, only small strain and linear elastic materials are taken into consideration. To know the displacement in the transverse direction (y-direction) where there is no actual loading on this direction, a virtual loading $Q$ is applied in the model and will be set as 0 in the later strain energy calculations. The applied loading, $P$, combined with a virtual loading, $Q$, can be divided into two components: longitudinal loading $P \cos \theta-Q \sin \theta$ along the length of the arm and perpendicular loading $P \sin \theta-Q \cos \theta$. The strain energy in longitudinal direction can be written as follows:

$$
\begin{aligned}
U_{l} & =\int_{0}^{l_{C u}} \frac{(P \cos \theta-Q \sin \theta)^{2}}{2 E_{C u} A_{C u}} d x \\
& =\frac{(P \cos \theta-Q \sin \theta)^{2} l_{C u}}{2 E_{C u} A_{C u}},
\end{aligned}
$$

where $A_{C u}$ is the cross-section area and $E_{C u}$ is the Young's modulus of the zigzag structure. The bending strain energy is as follows:

$$
\begin{aligned}
U_{M} & =\int_{0}^{l_{C u}} \frac{[(P \sin \theta+Q \cos \theta) \cdot x]^{2}}{2 E_{C u} I_{C u}} d x \\
& =\frac{(P \sin \theta+Q \cos \theta)^{2} l_{C u}^{3}}{6 E_{C u} I_{C u}},
\end{aligned}
$$

where $I_{C u}$ denotes the momentum of inertia, which is $I_{C u}=t_{C u} w_{C u}^{3} / 12$. The total strain energy combines both longitudinal tensile energy and bending energy. Therefore, the total energy can be written as follows:

$$
\begin{aligned}
U_{\text {total }}=U_{l} & +U_{M}=\frac{(P \cos \theta-Q \sin \theta)^{2} l_{C u}}{2 E_{C u} A_{C u}} \\
& +\frac{(P \sin \theta+Q \cos \theta)^{2} l_{C u}^{3}}{6 E_{C u} I_{C u}} .
\end{aligned}
$$

According to Castigliano's theorem, displacements can be obtained in each loading direction by $\delta_{P}=$ $\partial U_{\text {total }} / \partial P$ and $\delta_{Q}=\partial U_{\text {total }} / \partial Q$. Since $Q$ is a virtual loading, $Q=0$ has to apply in the calculations. Therefore, the displacements of one arm in the loading direction ( $x$-direction), $\delta_{x}=\delta_{P}$, and the virtual loading direction ( $y$-direction), $\delta_{y}=\delta_{Q}$, are as follows:

$$
\begin{gathered}
\delta_{x}=\frac{P l_{c u}}{E_{C u}}\left(\frac{\cos ^{2} \theta}{A_{C u}}+\frac{l_{C u}^{2} \sin ^{2} \theta}{3 I_{C u}}\right), \\
\delta_{y}=-\frac{P l_{C u}}{E_{C u}}\left(\frac{-\sin \theta \cos \theta}{A_{C u}}+\frac{l_{C u}^{2} \sin \theta \cos \theta}{3 I_{C u}}\right) .
\end{gathered}
$$

The total displacement in loading direction depends on the repeating number of arms, $n$, in the zigzag structure. Thus, the total displacement in loading direction is as follows:

$$
\delta_{x-\text { total }}=n \cdot \delta_{x}=n \cdot\left[\frac{P l_{C u}}{E_{C u}}\left(\frac{\cos ^{2} \theta}{t_{C u} w_{C u}}+\frac{4 \cdot l_{C u}^{2} \sin ^{2} \theta}{t_{c u} w_{C u}^{3}}\right)\right] \text {, }
$$

where $A_{C u}$ is replaced by $t_{C u} W_{C u}$. In addition, the total displacement in virtual loading direction, corresponding to the transverse direction, remains $\delta_{y}=\delta_{y \text {-total }}$.

In the free-body diagram, as shown in Fig. 2(b), the tensile stress in the arm caused by longitudinal loading is given by $\sigma_{l}=P \cos \theta / t_{C u} w_{C u}$. By combining the longitudinal tensile stress with the stress component caused by 
bending $\sigma_{M}=6 P l_{C u} \sin \theta / t_{C u} w_{C u}^{2}$, the maximal tensile stress $\left(\sigma_{\max }\right)$ in the arm is as follows:

$$
\sigma_{\max }=\frac{P \cos \theta}{t_{C u} w_{C u}}+\frac{6 P l_{C u} \sin \theta}{t_{C u} w_{C u}^{2}} .
$$

The stress distribution at the crest at $(x, y)=(0,0)$ in Fig. 2(a) is shown in Fig. 2(b). It is clear from this figure that this maximal tensile stress, which causes structural failure, occurs at the inner concave edge of the arm. Therefore, to prevent the structural failure, by increasing the width $\left(w_{C u}\right)$ or thickness $\left(t_{C u}\right)$, the tensile stress in the inner concave corner can be reduced. In other words, application-wise, the reliability of the zigzag interconnect can be improved. However, the stretchability, which equals to the total displacement in loading direction as shown in Eq. (6), reduces dramatically with increasing $w_{C u}$ and $t_{C u}$. Thus, the optimum design of this zigzag interconnect is an important challenge. This strategy is discussed in detail in our publication elsewhere.

The analytical models developed above only hold for small strain and linear elastic behavior because of Hook's law. However, in reality, a stretchable system is easily stretched more than $10 \%$, which causes large strain and plasticity in the stretchable interconnect. In addition, when a zigzag structure adhered on a polymer substrate is subjected to mechanical stretching, interfacial stress and peeling stress are generated. These effects are less easy to be implemented in analytical models. Therefore, the finite element method is used to simulate these complex behaviors. This process is discussed below in Sec. V. C.

\section{SAMPLE PREPARATION}

PDMS (Sylgard 186; Dow Corning, Midland, MI) was chosen as the elastomeric substrate, to carry a patterned metal on top. To achieve a stable resistance, low cost, and large area fabrication capability, we used a standalone $0.018-\mathrm{mm}$ thick commercially available copper foil. To improve the weak adhesion between the organic and the inorganic interface, a potassium monopersulfate solution was used for microetching the copper surface. The surface of the copper foil was $2 \mu \mathrm{m}$ in roughness as measured by an interferometer. The prepared copper foil was then temporarily adhered to a 0.5 -mm-thick Teflon mold, with an opening window for casting the PDMS on top of the copper foil. The PDMS was prepared at room temperature in a 10:1 weight ratio of the polymer base and curing agent. After degassing air bubbles, the PDMS was poured in the Teflon window and cured at $60{ }^{\circ} \mathrm{C}$ for $12 \mathrm{~h}$. Then, the $\mathrm{Cu} / \mathrm{PDMS}$ lamination was released from the Teflon mold and placed on top of the ceramic carrier. Both conventional photolithography and wet etching processes were used for patterning the zigzag structure. In the final step, the $\mathrm{Cu} / \mathrm{PDMS}$ lamination was cut

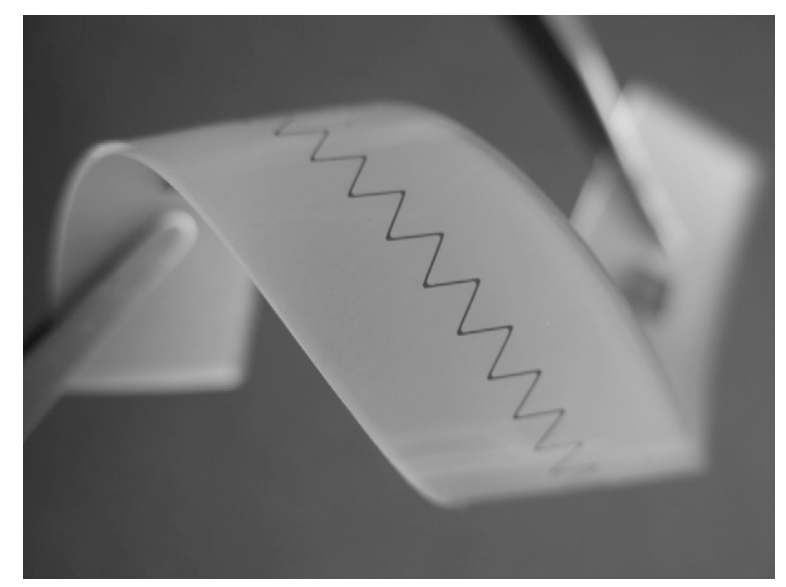

FIG. 3. Patterned zigzag interconnection with square pads for electrical connection.

into $10 \times 70$-mm strips. Each strip has only one zigzag interconnect composed of 42 crests. Figure 3 shows the flexibility of a completed zigzag interconnect sample.

\section{RESULT AND DISCUSSION}

The analytical models developed above consider the loading, $P$, as the applied boundary condition on the zigzag structure. By using this methodology, one can obtain the stress distribution as well as the deformation of the zigzag structure. However, in reality, the zigzag structure, even all kinds of metal interconnect, has to adhere to an elastomeric substrate, and thus the applied loading on the elastomeric substrate has to be taken into account in the analysis. Since the highly nonlinear behavior of the elastomeric substrate during large deformation, it is hard to predict the appropriate loading for a given elongation. Therefore, with the above-mentioned consideration and the nonlinearity on the stress-strain behavior of the elastomeric substrate, the following analysis both in numerical and experimental modeling will mainly use elongation as the boundary condition.

\section{A. In situ observation of substrate with zigzag interconnect subjected to uniaxial stretching}

The deformation processes and failure mechanisms of the zigzag interconnect subjected to a uniaxial stretching were investigated in situ by using a home-built set of tensile stage and fixture mounted directly in a JEOL 5600LV (Tokyo, Japan) scanning electron microscope (SEM). Figures 4(a) and 4(b) show the zigzag interconnect before stretching ( $0 \%$ strain) and during 60\% stretching. The whole zigzag interconnect is composed by 42 units following one after another. The two figures cover the same length in the stretching direction. It is clearly shown in Fig. 4(b) that the surface texture on the substrate changed compared with Fig. 4(a). This change on the surface texture was caused by sputtering carbon 


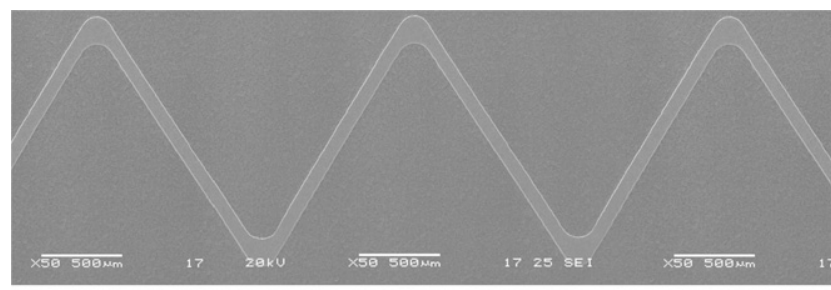

(a)

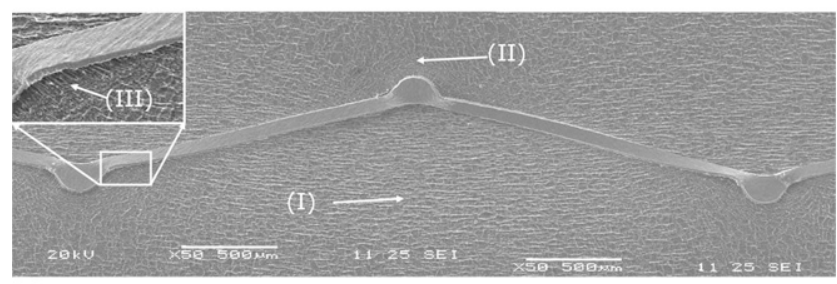

(b)

FIG. 4. Scanning electron micrographs of metallic zigzag-patterned interconnection (a) before stretching and (b) during stretching. The upper left inset of (b) shows a magnified view of the fibrillation.

on the same surface five times: before stretching, and after $25,40,50$, and $60 \%$ stretching. The purpose of five sputterings is to avoid charging of the substrate surface because the substrate material is not conductive. The sputtered carbon is not stretchable, and it is only a few nanometers thick; therefore, it is negligible in the overall strength/stiffness. By examining the surface texture in Fig. 4(b), three in-plane deformation mechanisms can be observed on the substrate surface:

(i) Uniaxial geometrical opening of the substrate in the stretching direction in the area $(I)$, evidenced by the rectangular shape of surface textures;

(ii) Negligible strain of the substrate in the area (II), identified by a curve-like texture circling the top of each crest;

(iii) Local shearing deformation in the area (III), observed by fiber-like surface texture near the interface (fibrillation).

The crest of the zigzag interconnect acts as the "rotation center" while the straight arms act as the radius. When stretching up to $60 \%$, as shown in Fig. 4(b), the zigzag interconnect deformed not only by in-plane geometrical opening and local twisting on the arms but also by out-of-plane deformation on the crests. The left column in Figs. 5(a)-5(d) shows a sequence of in situ observations on a stretched zigzag interconnect, starting from 25 up to $60 \%$ stretching. These micrographs attest the three deformation mechanisms mentioned above. Meanwhile, these micrographs also show a system in which the degree of stretchability is not determined simply by the overall geometrical opening of the zigzag interconnect. Moreover, the local in-plane shear strain on the substrate, mentioned above in the third deformation mechanism, is responsible for a local sliding deformation. This local sliding deformation induced by a local fibrillation process contributes parts of the overall stretchability. It is noted that this sliding deformation induced by the fibrillation process can be elastically recovered once the zigzag structure released back to its original position. This deformation mechanism can be explained by the elastic nature of substrate's material properties because the fibrillation process only occurred on elastomeric substrate. Moreover, one can also observe that the fibrillation is accompanied by or "followed by" the interfacial delamination. Once the fiberlike texture no longer holds the zigzag structure sliding on the substrate surface, the interfacial delamination starts propagating at the interface. The right column of Fig. 5 shows the contours of in-plane shear strain in the substrate obtained by FEM analysis corresponding to the left column of the in situ observations. The contours of in-plane shear strains in the substrate have the maximal values near the free edge of the zigzag interconnect close to each crest. These in-plane shear strain contours confirm that crests work as the center and the arms work as the radius. As the zigzag interconnect is being stretched, the arms rotate and the crests remain, resulting in a localized in-plane shear strain concentration close to each crest.

\section{B. Failure analysis of the zigzag interconnect}

To observe the failure mechanism of the $\mathrm{Cu}$ interconnect, the home-built tensile stage that was mounted directly in the SEM was tilted 45 degrees and rotated with appropriate angle, as shown in Figs. 6(a) and 6(b). Although there is an out-of-plane deformation on the crest during $40 \%$ stretching, as shown in Fig. 6(a), the crest is still adhered on the substrate surface without delamination. This feature is possible because the substrate is sufficiently compliant. This deformation mechanism, which is different from the one for a freestanding zigzag interconnect, results in localized strain. Consequently, local compressive strain is concentrated at the outer convex edge of the zigzag interconnect close to the crest; meanwhile, local tensile strain is concentrated at the inner concave edge of the zigzag interconnect opposite to the compressive concentration location. It is obvious in Fig. 6(b) that there is severe strain concentration when stretching up to $60 \%$, creating possible failure locations when the local strain concentration becomes higher than the strain limit of the involved materials. Besides strain concentration on the arms, the crest has a significant out-of-plane deformation while it remains adhered to the substrate surface. It is noted that the interface between $\mathrm{Cu}$ and PDMS no longer shows an intact adhesion but is subject to fibrillation. This fibrillation process at the crest is mainly driven by interfacial normal stress or "peeling stress." The fibrillation process was also observed at 

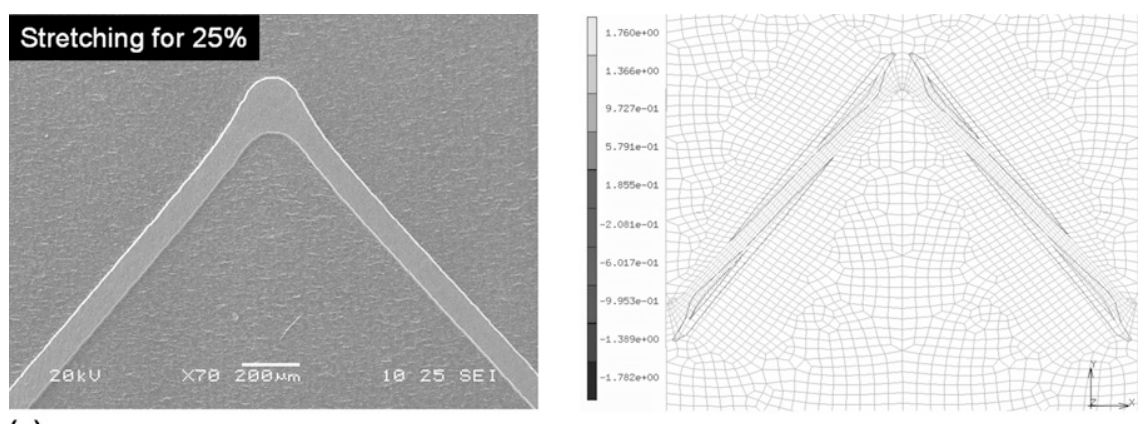

(a)
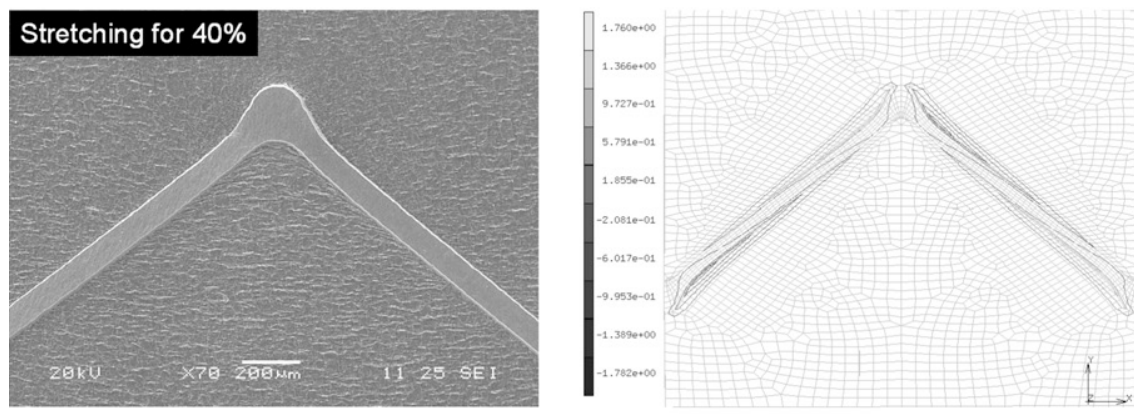

(b)
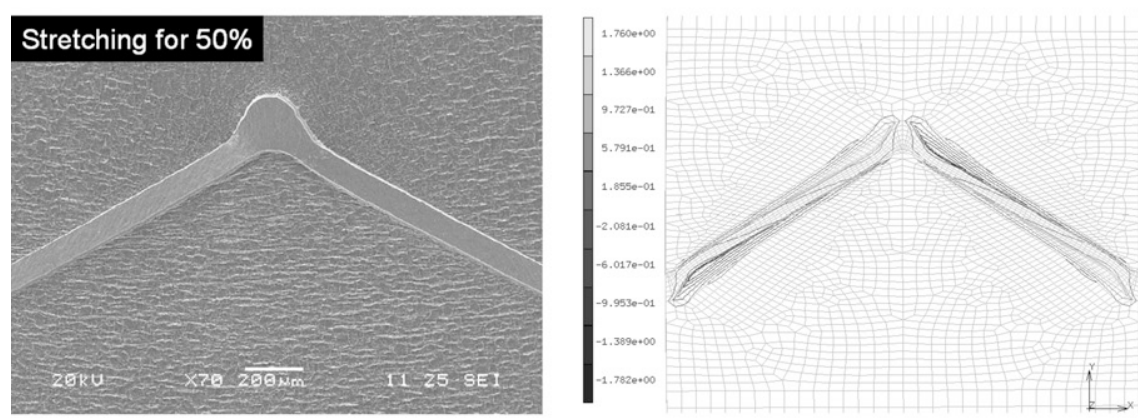

(c)
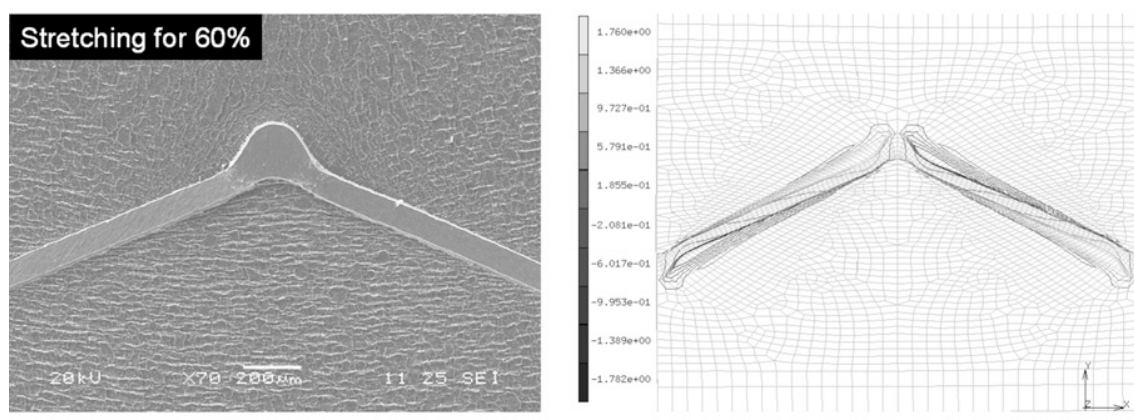

(d)

FIG. 5. Micrographs in the left column were taken in situ while stretching for (a) $25 \%$, (b) 40\%, (c) 50\%, and (d) 60\%; in-plane shear strain contours $\left(\varepsilon_{\mathrm{xy}}\right)$ of the PDMS substrate in the right column correspond to the left micrographs. Only FE meshes of PDMS substrate are shown.

the interface of the arms when stretching up to $60 \%$. This result is due to the interfacial shear stress when the zigzag interconnect is subjected to external loading. The failure modes such as fibrillation and delamination, induced by these two interfacial stresses, are extremely sensitive to the bonding strength between the metal and the substrate. Therefore, these two interfacial stresses combined with geometrical opening are respon- sible for both stretchability and reliability, and they will be discussed in detail under Sec. C.

Figures 7(a) and 7(b) show the breakdown mechanisms of the zigzag interconnect when stretched for $62 \%$. These images are taken once the failure was observed, and the zigzag interconnect was released back to its original position to prevent further delamination. It is clearly shown that, in Fig. 7(a), the crest had in-plane 


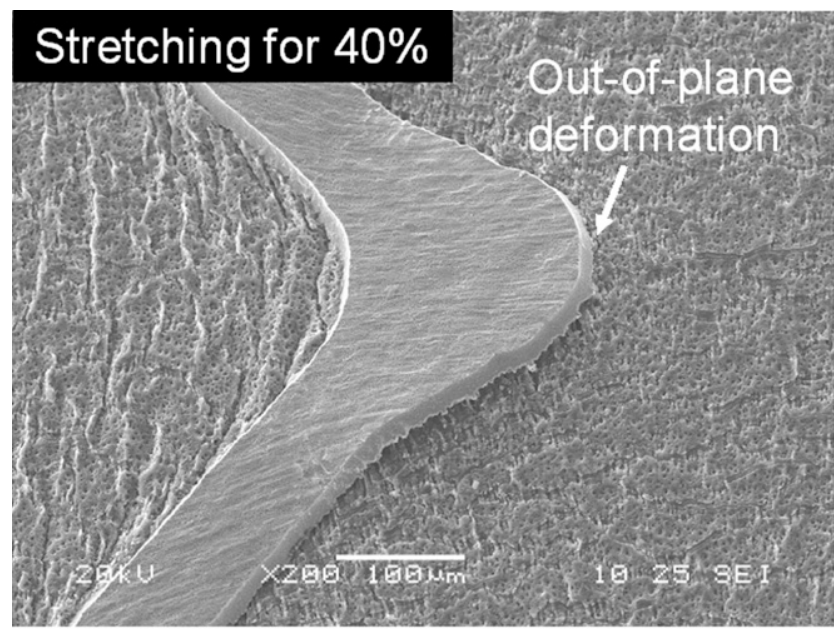

(a)

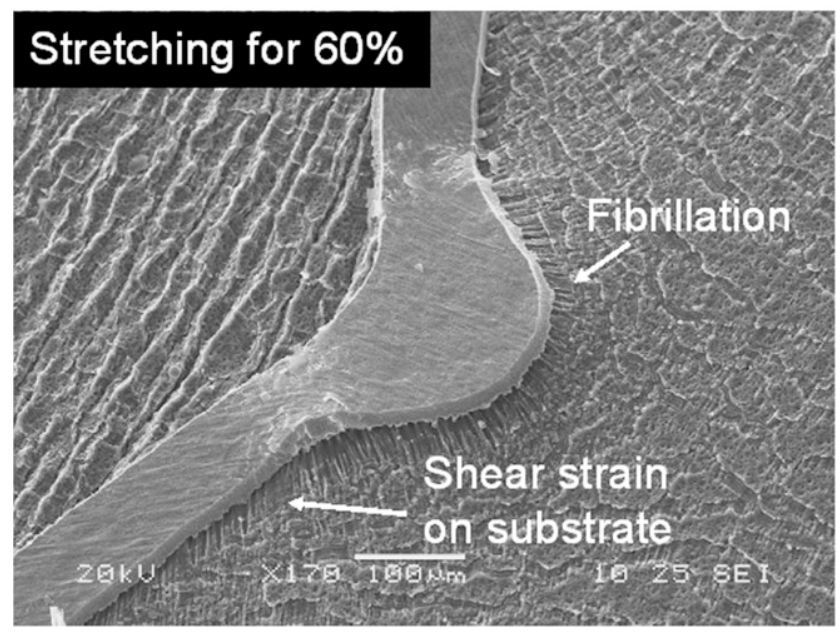

(b)

FIG. 6. The zigzag interconnection was rotated and tilted in the scanning electron microscope: (a) stretched for $40 \%$; (b) stretching for $60 \%$.

plastic deformation, and, furthermore, in Fig. 7(b), the crest plastically deformed out-of-plane without elastically returning back to its original position. It is noted that not only are there twisting arms, delamination at the interface, and plastic deformation on the crest, but also the metal broke and therefore caused cratering in the substrate at the same location. The whole zigzag interconnect was stretched up to $62 \%$, which caused the metal breakdown; however, there is no significant necking observed at the cross-section of the metal rupture area. It is suspected that when the zigzag interconnect is being stretched to a large deformation, the rate of strain is not uniformly distributed along the zigzag structure. Instead, the junction between crest and arm has locally high rate of strain because the deformation mechanism is mainly the geometrical opening. This locally high rate of strain, combined with the locally complex combination of stress state and small feature

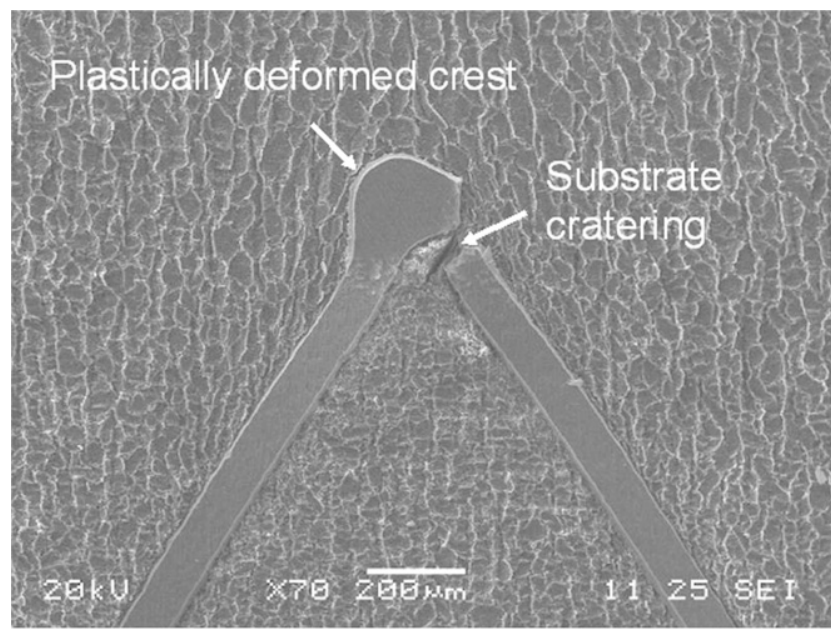

(a)

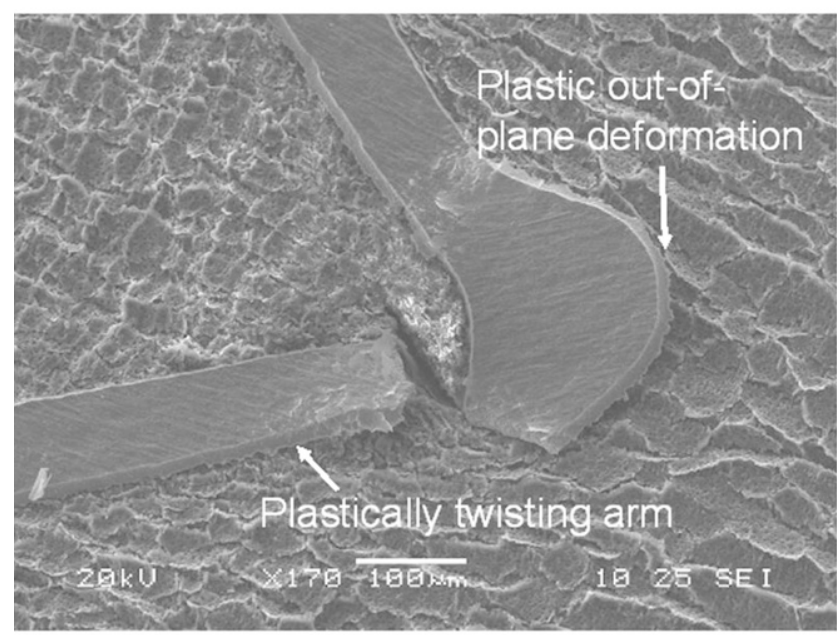

(b)

FIG. 7. Typical breakdown mechanism of zigzag interconnection. (a) Released state to see the plastic deformation; (b) rotated and tilted the zigzag interconnection.

size of zigzag structure, may result in a transition from ductile behavior to brittle behavior. Therefore, the local plastic necking on the arm-to-crest junction of zigzag structure is not as significant as the necking in bulk metal under tensile test. This phenomenon was also observed and attested by the resistance measurements in our previous publication. ${ }^{15}$ If there were significant plastic necking during stretching, the resistance would increase substantially before failure instead of remaining constant. Figure 8 illustrates the typical deformation pattern of the arms and the crest on the repeating unit of the zigzag structure after stretching. The plastically deformed twisting arms caused local delamination from the substrate in one edge and compression on the PDMS substrate in the other. The crest was plastically deformed out-of-plane from the substrate, and the fibrillation was not observed because the substrate elastically returned back to its original position. 


\section{Mechanical modeling}

All of the observations described above can be understood and quantified by using numerical modeling. Figure 9(a) illustrates the FEM model in the relaxed

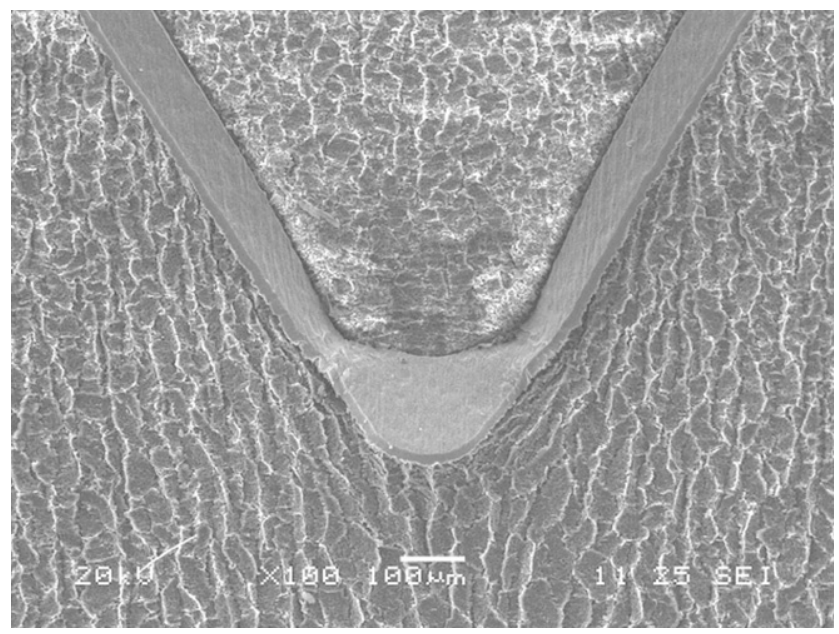

FIG. 8. A typical deformation pattern of a repeating unit after stretching.

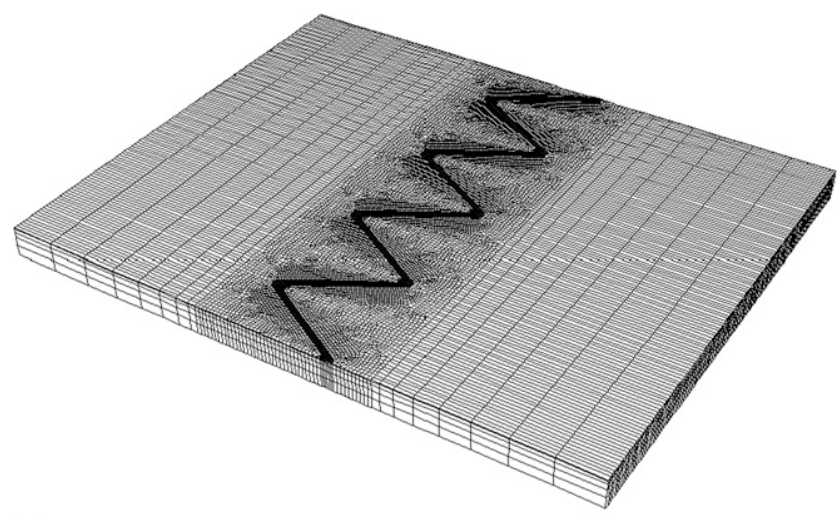

(a)

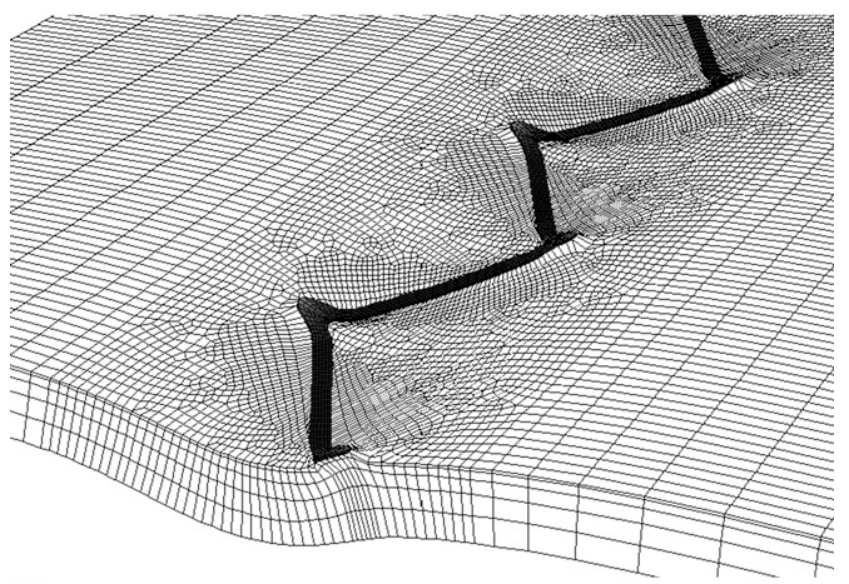

(b)

FIG. 9. (a) Finite element meshes before stretching; (b) cross-section view of out-of-plane deformed zigzag interconnection while stretching for $60 \%$. state, in which the geometry corresponds to the middle block of the real zigzag interconnect in the experiment. During displacement $(\mu)$ at the two end surfaces of up to $60 \%$ strain, both the middle portion of the substrate where the zigzag interconnect resides as well as the zigzag interconnect deformed out-of-plane in concave shape. These concave shaped and out-of-plane deformations are shown in Fig. 9(b). It is clearly shown in Figs. 9(b) and 10 that there are two major deformation mechanisms involved in the stretching process: out-of-plane deformation and geometrical opening. These two major deformation mechanisms are explained by two sections of the curve in Fig. 11. Figure 11 plots the maximal out-of-plane deformation of the zigzag interconnect as a function of the relative elongation of the substrate. To avoid confusion with the strain in the zigzag interconnect, the relative

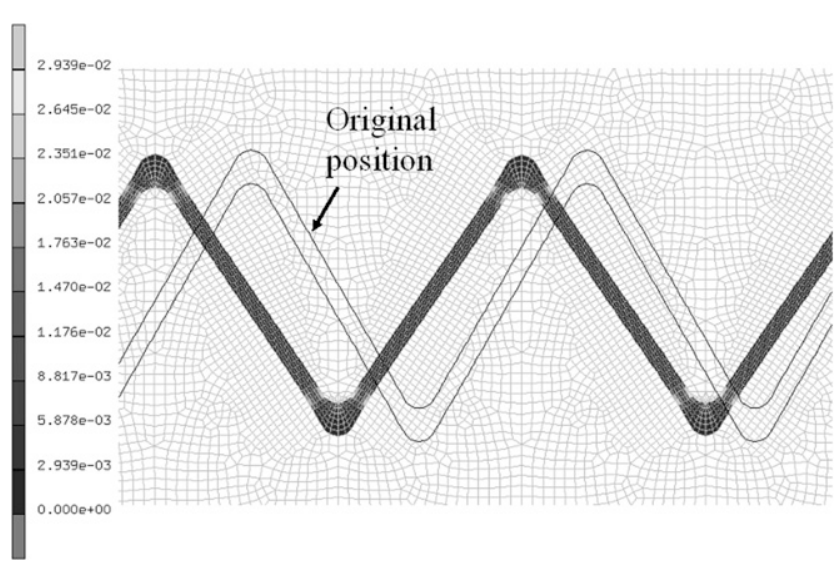

FIG. 10. Geometrical opening and plastic strain distribution on deformed zigzag interconnection. Black lines show the original position.

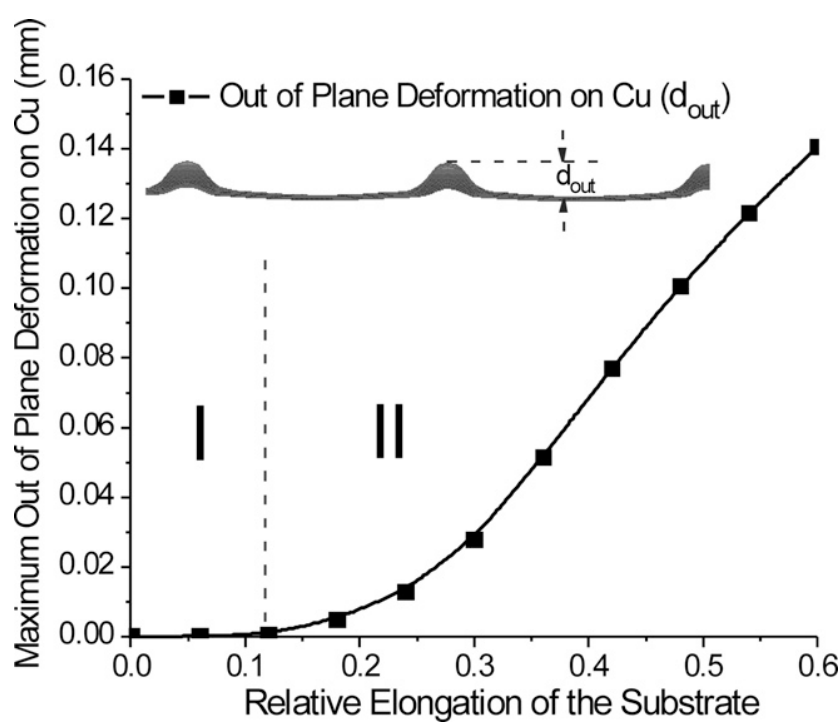

FIG. 11. Maximal out-of-plane deformation $\left(d_{\text {out }}\right)$ of zigzag interconnection as a function of the relative elongation of the substrate. The inset is a cross-section view showing the definition of $d_{\text {out }}$ on finite element (FE) meshes of the zigzag interconnection. 
elongation of the substrate is expressed as $\mu / L_{\text {sub }}$. In Fig. 11, the maximal out-of-plane deformation of the zigzag interconnect occurs on its outer convex edges of the crests. As the relative elongation of the substrate increases from 0 to $12 \%$ in Sec. I, the maximal out-of-plane deformation of the zigzag interconnect increases only slightly. The major deformation mechanism in this stage is mainly in-plane geometrical opening, initiating strain concentration on the arms. This process is seen as local tensile strain concentration at the inner concave edge of the arms and local compressive strain concentration at the outer convex edge of the arms. Furthermore, as the relative elongation increases from 12 to $60 \%$, in Sec. II, the maximal out-ofplane deformation of the zigzag interconnect increases substantially. The substrate is no longer able to keep the zigzag interconnect deforming in the same plane. Instead, out-of-plane deformation takes place to accommodate the further stretching loading, so that even a large elongation induces only small strain in the zigzag interconnect.

Figure 10 not only shows the geometrical opening but also the plastic strain distribution on the deformed zigzag interconnect. Plastic strain keeps accumulating on the inner concave edge of the arms during stretching, and, consequently, the metal breaks down. Referring back to Fig. 7, it is found that the strain distribution on the zigzag interconnect reached by numerical analysis agrees well with the failure location observed experimentally. Moreover, this result corresponds with the location of maximal stress concentration in our analytical models.

Figure 12 shows both the maximal in-plane strain in the substrate and the equivalent plastic strain in the $\mathrm{Cu}$ interconnect as functions of the relative elongation of the substrate, as derived from finite element simulation. The plastic strain in the metal is minimized to avoid the metal failure during stretching. In the zigzag-patterned design, the maximal plastic strain in the lines increases nearly linearly up to $8.1 \%$ at a relative elongation of $60 \%$. Maximal in-plane strain in the substrate occurred on its surface near the $\mathrm{Cu}$ arms and close to the $\mathrm{Cu}$ crests, with a magnitude much higher than that of the applied relative elongation. This result is because the arms rotated while stretching and induced local distortion of the substrate near the arms. Figure 13 plots both the maximal interfacial peeling stress and maximal interfacial shear stress as functions of the relative elongation of the substrate. The interfacial peeling stress can be negligible from 0 to $12 \%$ relative elongation of the substrate, because the deformation mechanism is mainly an in-plane geometrical opening, as explained in Fig. 11. When the relative elongation of the substrate goes above $12 \%$, the interfacial peeing stress increases due to the out-of-plane deformation of the crests. Even though the relative elongation of the substrate reached $60 \%$, the interfacial peeling stress barely increased. This phenom-

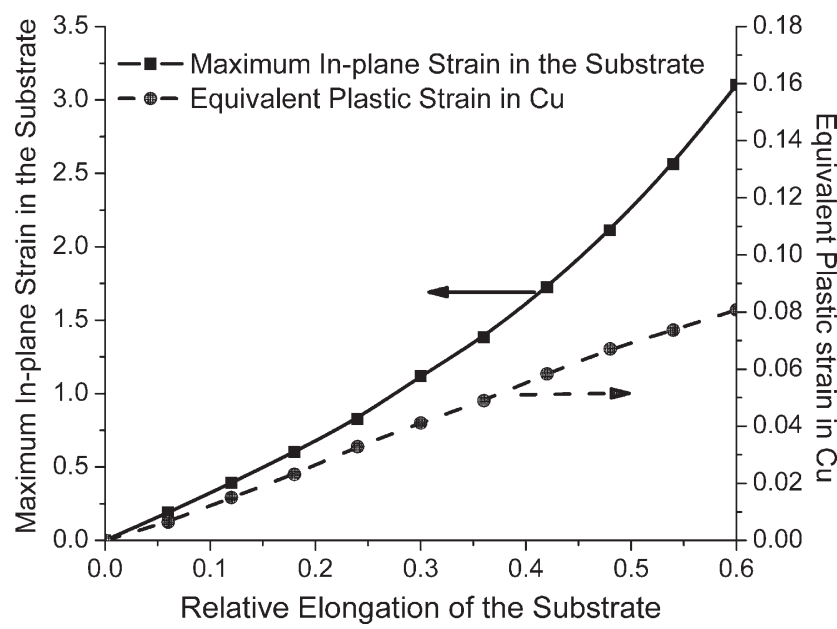

FIG. 12. The maximal in-plane strain in PDMS substrate and the equivalent plastic strain in $\mathrm{Cu}$ zigzag interconnection as functions of the relative elongation of the substrate.

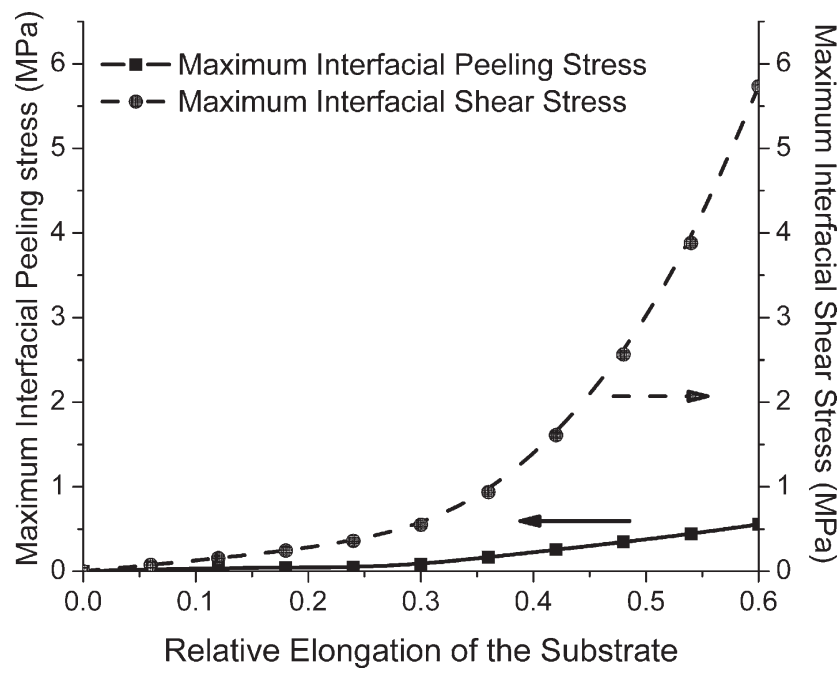

FIG. 13. The maximal interfacial peeling stress and the maximal interfacial shear stress as functions of relative elongation of the substrate.

enon is explained by the highly compliant substrate used in this research. Compared with the maximal interfacial peeling stress, the maximal interfacial shear stress increases substantially, because the relative elongation of the substrate increases. This phenomenon suggests that the interfacial shear stress dominates the failure and the reliability of the stretchable interconnect.

\section{CONCLUSIONS}

In summary, a zigzag-patterned interconnect adhering to an elastomeric substrate was stretched and observed in situ in a scanning electron microscope. The deformation behaviors and failure mechanisms were identified and correlated to numerical simulations to understand the inherent mechanics. Our experiments showed that the 
zigzag interconnect can be stretched up to $62 \%$ while experiencing three different failure modes: fibrillation of the substrate near the edge of the arms, out-of-plane deformation of the crest, and breaking on the metal arms. These failure modes were governed by interfacial in-plane shear stress in the substrate, interfacial peeling stress, and local plastic strain in the metal, respectively. The interfacial in-plane shear stress dominated failure and stretchability compared with the interfacial peeling stress. Nevertheless, there was a significant out-of-plane deformation, confirmed both by numerical and experimental results. By examining the metal breakdown area, it was found that there was no significant local plastic necking. This observation was also confirmed by electrical resistance measurements in our previous publication. ${ }^{15}$

It is also observed through analytical analysis that the stretchability highly depends on the zigzag structure design. Based on the analytical model in this article and numerical analysis, ${ }^{11,14}$ it is suggested by choosing a compliant, thin substrate, and high length-to-width ratio of the arm, soft metal, the high stretchability zigzag interconnect can be realized.

The above analysis and results show that the zigzag interconnect offers promising possibilities for stretchable interconnects, especially for fine pitch applications. In the future, further studies will focus on metal ductility, interfacial separation energy, and the mechanics of cohesive cracking. This approach will allow us to further understand, and to improve through design, the stretchability and reliability of this zigzag interconnect.

\section{ACKNOWLEDGMENT}

This work was supported by European Commission, under the STELLA research project (Contract No. 028026).

\section{REFERENCES}

1. V.J. Lumelsky, M.S. Shur, and S. Wagner: Sensitive skin. IEEE Sensors J. 1, 41 (2001).

2. S. Wagner, E. Bonderover, W.B. Jordan, and J.C. Sturm: Electrotextiles: Concepts and challenges. Int. J. High Speed Electron. Svst. 12, 1 (2002).
3. H. Jiang, D.Y. Khang, J. Song, Y. Sun, Y. Huang, and J.A. Rogers: Finite deformation mechanics in buckled thin films on compliant supports. Proc. Nat. Acad. Sci. U.S.A. 104(40), 15607 (2007).

4. H. Jiang, Y. Sun, J.A. Rogers, and Y. Huang: Mechanics of precisely controlled thin film buckling on elastomeric substrate. Appl. Phvs. Lett. 90, 133119 (2007).

5. H.C. Ko, M.P. Stoykovich, J. Song, V. Malyarchuk, W.M. Choi, C.J. Yu, J.B. Geddes, J. Xiao, S. Wang, Y. Huang, and J.A. Rogers: A hemispherical electronic eye camera based on compressible silicon optoelectronics. Nature 454, 748 (2008).

6. D.H. Kim, J.H. Ahn, W.M. Choi, H.S. Kim, T.H. Kim, J. Song, Y.Y. Huang, L. Zhuangjian, L. Chun, and J.A. Rogers: Stretchable and foldable silicon integrated circuits. Science 320, 507 $\underline{(2008)}$.

7. J.H. Ahn, H.S. Kim, E. Menard, K.J. Lee, Z. Zhu, D.H. Kim, R.G. Nuzzo, J.A. Rogers, I. Amlani, V. Kushner, S.G. Thomas, and T. Duenas: Bendable integrated circuits on plastic substrates by use of printed ribbons of single-crystalline silicon. Appl. Phvs. Lett. 90, 213501 (2007).

8. S.P. Lacour, S. Wagner, Z. Huang, and Z. Suo: Stretchable gold conductors on elastomeric substrates. Appl. Phvs. Lett. 82(15). 2404 (2003).

9. S. Wagner, S.P. Lacour, J. Jones, P.I. Hsu, J.C. Sturm, T. Li, and Z. Suo: Electronic skin: Architecture and components. Phvsica E 25. 326 (2004).

10. T. Li, Z. Huang, Z. Suo, S.P. Lacour, and S. Wagner: Stretchability of thin metal films on elastomer substrates. Appl. Phvs. Lett. 85(16), 3435 (2004).

11. T. Li, Z. Suo, S.P. Lacour, and S. Wagner: Compliant thin film patterns of stiff materials as platforms for stretchable electronics. J. Mater. Res. 20(12), 3274 (2005).

12. D.S. Gray, J. Tien, and C.S. Chen: High-conductive elastomeric electronics. Adv. Mater. 16(5), 393 (2004).

13. D. Brosteaux, F. Axisa, M. Gonzalez, and J. Vanfleteren: Design and fabrication of elastic interconnections for stretchable electronic circuits. IEEE Electron Device Lett. 28(7), 552 (2007).

14. M. Gonzalez, F. Axisa, M. Vanden Bulcke, D. Brosteaux, B. Vandevelde, and J. Vanfleteren: Design of metal interconnects for stretchable electronic circuits. Microelectron. Reliab. 48, 825 (2008).

15. Y.Y. Hsu, M. Gonzalez, F. Bossuyt, F. Axisa, J. Vanfleteren, and I. DeWolf: A novel interconnect design with high stretchability and fine pitch capability in applications of stretchable electronics. Mater. Res. Soc. Symp. Proc. (2009).

16. S.L. Chiu, J. Leu, and P.S. Ho: Fracture of metal-polymer line structures. I: Semiflexible polyimide. J.Appl. Phvs. 76(9), 5136 (1994).

17. MSC Marc User Manual. 